\title{
Management of amiodarone induced thyrotoxicosis within the United Kingdom: is it time for a consensus guideline? A single centre retrospective review
}

Mie Mie Tisdale, Francesca Swords, Rupa Ahluwalia

Department of Diabetes and Endocrinology, Norfolk and Norwich University Hospitals NHS trust, Norwich, United Kingdom

\section{Introduction}

Amiodarone induced thyrotoxicosis (AIT) remains a diagnostic and therapeutic challenge.

AIT is classified as

type 1 (underlying latent thyroid disorder)

$>$ type 2 (destructive thyroiditis).

Type 1 and 2 are treated differently however, in many cases, the precise aetiology of AIT is unclear at the time of presentation.

There is currently no UK wide guidance on management of AIT

We present a retrospective review of consecutive cases treated for AIT in our department over 4 years.

\section{Methods}

Audit study period : 2010 - 2014

Thirty-nine patients presenting with AIT were studied, with complete data available on thirty-three.

Key audit data

$>$ Duration of amiodarone therapy prior to diagnosis

$>$ Type of AIT

$>$ Thyroid peroxidase antibody (TPO) levels

$>$ Imaging

Time to remission of AIT

$>$ Treatment strategy

\section{Results}

Age : Average age 66 years(range :30 - 81 years)

Sex: Predominantly men (79\%)

Duration of amiodarone therapy : Average 45 months

(range: 8 - 132 months)

$$
\begin{array}{cc}
\text { TPO status : TPO positive } & 3 \text { patients }(9 \%) \\
\text { TPO negative } & 24 \text { patients }(73 \%) \\
\text { TPO status not checked } 6 \text { patients }(18 \%)
\end{array}
$$

Amiodarone was discontinued in $28(85 \%)$ patients

Two patients underwent emergency thyroidectomy post medical treatment while only one patient was referred for radioiodine (RAI) ablation.

No significant difference in time to remission with or without Amiodarone therapy (3 vs. 2.8 months).

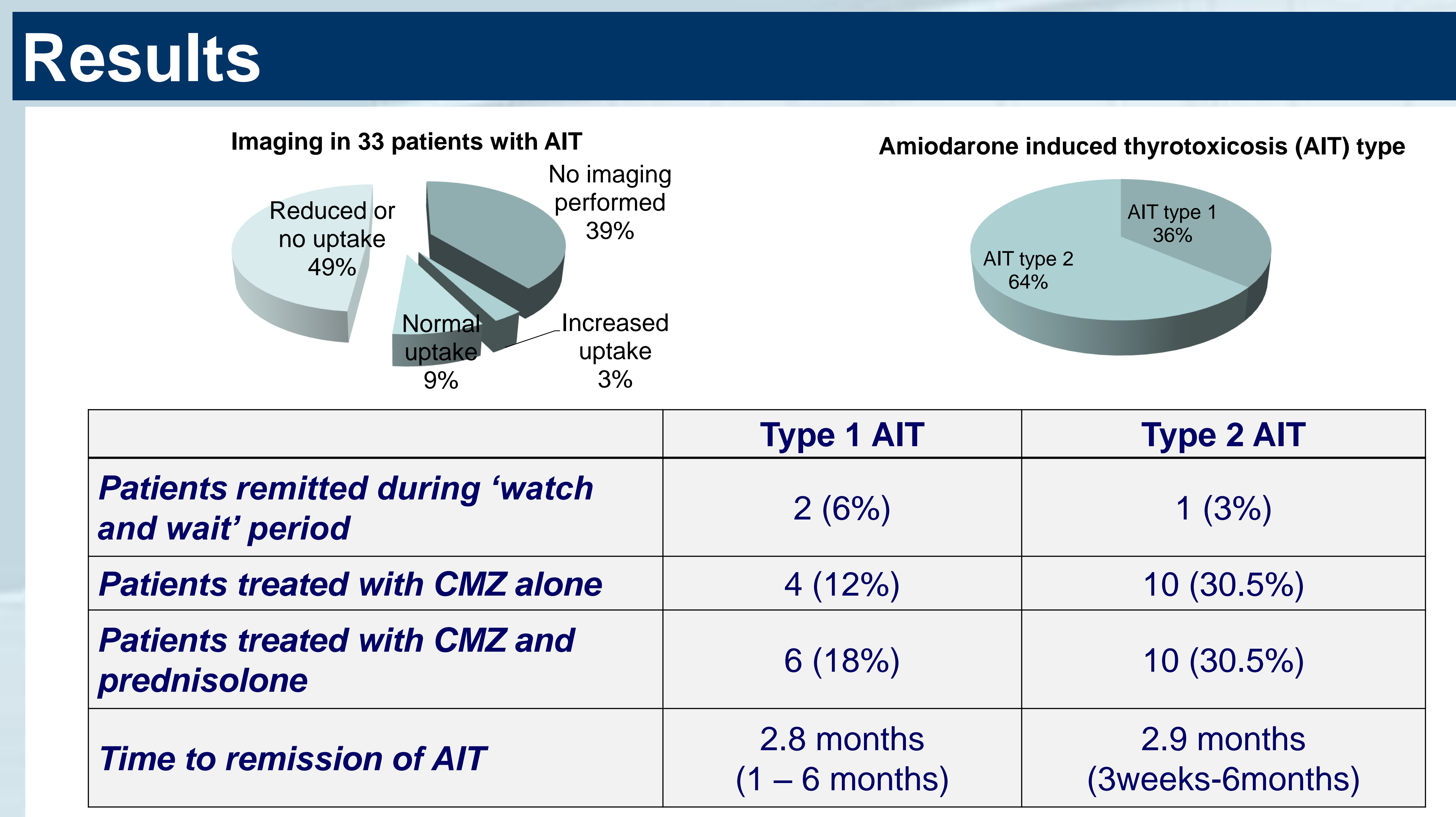

\section{Conclusions}

- In the absence of clear guidelines for classification and management of both types of AIT, overall practise remains primarily determined by individual clinician's experience as highlighted in surveys undertaken by both the European Thyroid association ${ }^{1}$ and within the United Kingdom ${ }^{2}$.

$\circ$ Our review further highlights differences in practise amongst endocrinologists in the diagnosis and management of AIT.

- Based on our audit findings, we propose a practical and evidence-base guide in management of AIT.

1.Bartalena et al. Clin Endocinol (oxf). 2004 Oct;61(4);494-502

2.Raghavan et al. Clin Endocinol (oxf). 2012 Dec;77(6):936-7

\section{Recommendations}

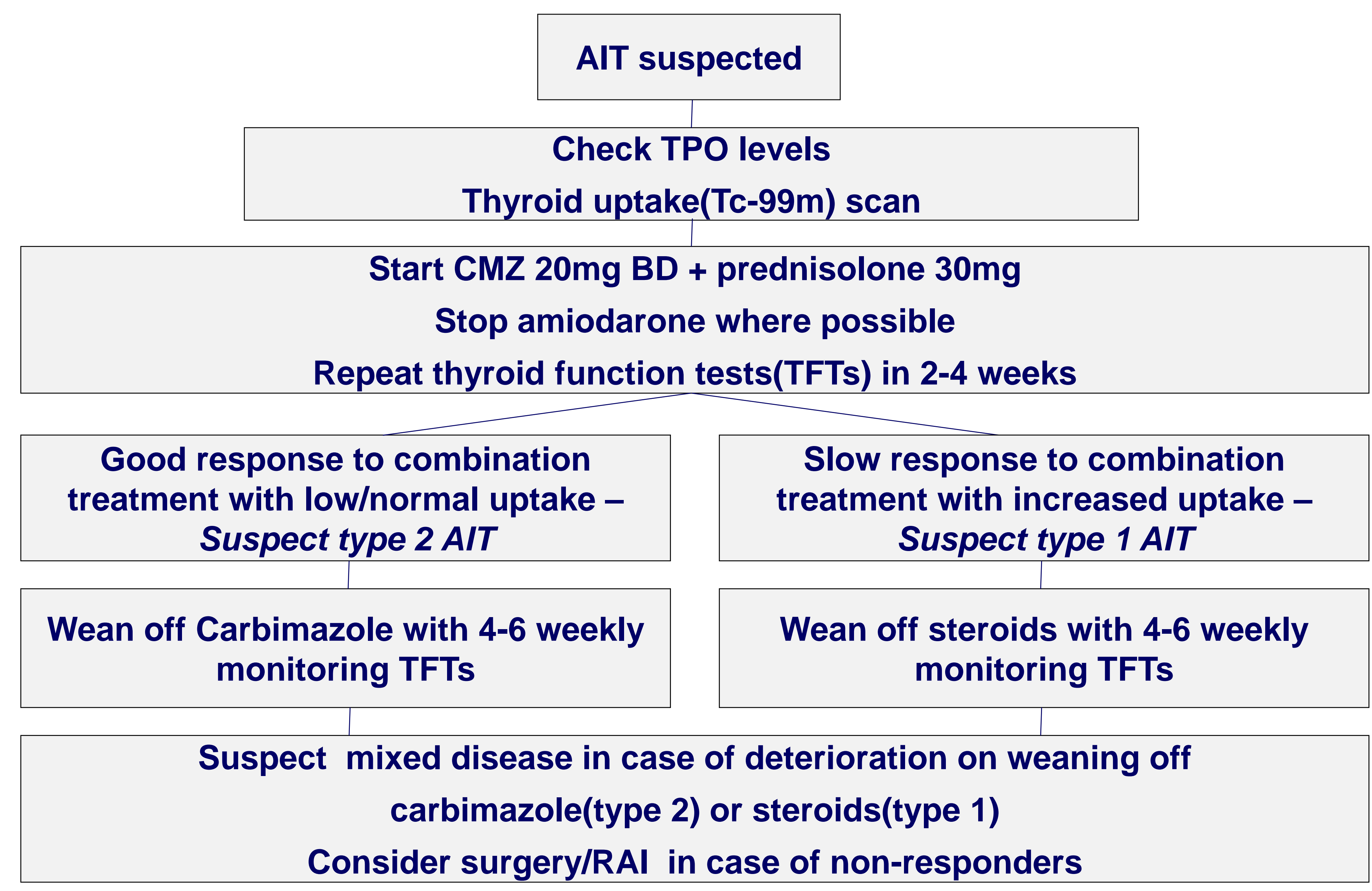

